\title{
Audit: Patient reported outcomes of extracorporeal septorhinoplasty*
}

\author{
D. Mendis', P. P. Cheang 2 , L. P. Glossop ${ }^{1,2}$
}

' Department of ENT Surgery, New Cross Hospital NHS Trust, Wednesfield Road, Wolverhampton WV10 0QP, United Kingdom

2 Deartment of ENT Surgery, Russell's Hall Hospital NHS Trust, Dudley DY1 2HQ, United Kingdom
Rhinology 51: 000-000, 2013 DOl:10.4193/Rhino12.085

*Received for publication: May 21, 2012

Accepted: January 17, 2013

\section{Summary}

Background: Extracorporeal septorhinoplasty (ECS) presents an important option in the surgical correction of the markedly deformed and twisted nose. However, there is a paucity of literature on the qualitative outcomes of ECS.

Methodology: Retrospective telephone and postal questionnaire study of operations performed over 2 years (2008-2009) under the care of a senior rhinologist at two linked hospitals in the United Kingdom.

Questionnaires were designed (i) relating to the procedure and (ii) patient perceptions of surgical success, improvements in physical health, psychosocial functioning and social interactions. For succinctness a shortened version of the Glasgow Benefit Inventory was used.

Results: Forty-six cases were analysed eight of which were revision cases. Two cases required revision surgery and two minor complications occurred, managed conservatively. The patients had a minimum of two to three follow-ups from seven days to one year. Questionnaire scores suggested high satisfaction in response to surgical success, with notable improvements in physical health (sleep disturbance) and in general health and well-being. Regarding psychosocial functions patients reported feeling either moderately less self-conscious or not self-conscious at all post-surgery. Improvements in social interactions were less well-defined.

Conclusion: In this audit of cases with significant deformities, ECS appears to confer high levels of satisfaction.

Key words: audit, extracorporeal septorhinoplasty, foundation tripod, Glasgow Benefit Inventory, septal cartilage

\section{Introduction}

Patient reported outcomes have become an important aspect in validating health care interventions both for research to establish, which treatment is effective and for clinical governance and audit.

Septorhinoplasty aims to improve quality of life. The Glasgow Benefit Inventory (GBI) is a patient-orientated questionnaire which assesses post-interventional change in health status and was felt to be the most appropriate assessment tool for this procedure, being a measure of physical, social and psychological wellbeing ${ }^{(1)}$.

The shape and projection of the cartilaginous portion of the foundation tripod is influenced by the integrity of the septal and upper lateral cartilages and crucially, by the rigidity of the 
junction between these cartilages. Simple nasal deviations may be dealt with endonasally but this approach does not permit access to the cephalic edge of the septal cartilage and thus cannot influence the shape of the twisted or deviated nose.

Removal and reinsertion of the intact septal cartilage was first described in the 1950s for severe septal deformities ${ }^{(2)}$. Rees later described a method for correction of the deviation involving total removal and reversal of the septal cartilage naming this technique an extracorporeal septoplasty (ECS) ${ }^{(3)}$.

Significant challenges associated with this method are ensuring adequate stabilisation of the septal cartilage graft and prevention of dorsal irregularities. To address this Gubisch (1995) described, in a series of 1012 cases suture fixation of the septal cartilage graft to the tip and base of the columellar and to the nasal spine but noted dorsal irregularities at a frequency of $8 \%$ (4).

The extracorporeal management of the septum has gained acceptance in septorhinoplasty for the twisted nose in which the dislocation or distortion of the septal cartilage has deformed the foundation tripod and, in many cases, affected the form and position of the tip. Its advantage lies in the facility to reshape and trim the cartilage, with battens if appropriate thus retaining its integrity, returning it to the midline on a prepared maxillary crest. The foundation tripod can then be reconstructed on this central support.

Scanty research exists on the patient reported outcomes of ECS. Our study is an audit based on the outcomes of extracorporeal septorhinoplasty performed at two linked hospitals

\section{Materials and methods}

Multicentre study conducted at two-linked West Midlands teaching hospitals (Russell's Hall Hospital, Dudley and New Cross Hospital, Wolverhampton, UK) analysing the operative outcomes of ECS performed or supervised by the senior author over a 2-year period (2008-2009). Retrospective in design, cases were identified by use of the information systems and audit department at both sites by using the relevant Office of Population Censuses and Surveys Classification of Interventions and Procedures version 4 (OPCS) code. Cases were cross-matched with theatre operative logbooks to pick-up additional cases. Cases which were not ECS (conventional closed or open septorhinoplasty) were excluded.

Note should be given to this being a heterogenous group related to both indications for surgery and the absence of a consistent representative system for describing and scoring deformity, making it difficult to offer individual advice to an individual patient.

In relation to data collection, two proformas were devised to collect (i) quantitative data for collection of demographic and operative details, follow-up and complications from medical notes; (ii) and semi-qualitative data via a questionnaire where respondents were asked to select a response based on a 5-point Likert scale based on four domains of surgical success, physical health, psychosocial function and social interactions.

As this study was intended as an audit the semi-qualitative data was collected by a questionnaire which extracts some of the questions based on the Glasgow Benefit Inventory (GBI) consisting of nine questions as opposed to the full eighteen. This was done because data collection was intended to be initially by telephone and so for the purposes of succinctness a revised questionnaire was devised. When patients were unobtainable via the telephone on two occasions questionnaires were posted. Written and verbal consent was obtained in both instances. Robustness and consistency of data collection was encouraged by the use of two senior trainee auditors (one at each site).

\section{Ethical considerations}

Consent was obtained from patients verbally for those questionnaires completed by telephone and via a letter for postal questionnaires.

\section{Bias}

The authors recognise possible sources of bias as being selection bias (inherent in any postal questionnaire survey) and recall bias.

\section{Surgical methods}

Surgery was performed under general anaesthetic with a throat pack. Lignocaine $2 \%$ with adrenaline 1 in 80,000 was infiltrated into the septum, columellar, tip and dorsum and into two areas on the sidewalls, the sites of percutaneous osteotomies. An open approach was used and the lower flap on the columellar was raised to expose the nasal spine. The medial crurae were separated and the membranous septum dissected to approach the septal cartilage. Both mucoperichondrial flaps were raised widely and the cartilaginous septum dissected from the maxillary crest, vomer and perpendicular plate. Using a scalpel, the septal cartilage was separated flush from the upper lateral cartilages but leaving the upper laterals attached to one another thus retaining the "spreading" arrangement and a firm bed for grafts if required. The removal of one element of this three-point junction created a degree of plasticity, allowing the replaced septum to influence its shape and position.

Prior to the re-insertion of the septal cartilage, any required 
bone work was undertaken. The septal cartilage was then prepared. The minimum requirement was a roughly rhomboid, flat piece to sit on the prepared crest and extend superiorly to support the middle third of the dorsum and tuck under the "keystone" area. Two-point fixation of the cartilage was achieved by an anterior suture encircling or inserted into the periosteum of the nasal spine and a superior suture attaching the septum to the upper lateral cartilages using polydioxanone suture (PDS). Trimming usually generated enough spare cartilage to use as a baton to straighten an angulation or curve without loss of strength and to use for supratip grafting and a columellar strut.

Tip work often involved the modification of an asymmetrical or boxy tip. We adopted the principle of suturing rather than resection where at all possible. Where tip projection was effected, wide release of the inferior columellar flap was undertaken to avoid tension and tip droop.

Internal incisions were closed with absorbable sutures, external with 5.0 nylon. A thermoplastic splint was applied externally and dissolvable sponge (Nasopore ${ }^{\circledR}$ ) packs intranasally.

\section{Results}

\section{Demographics and summary of operative details}

- 92 cases were identified initially and finally 46 cases analysed with a complete data set.

- Gender ratio consisted of 28 Male: 18 Female (61\% versus 39\%)

- Outcome questionnaires were completed by telephone $48 \%$ cases versus postal $52 \%$. Postal questionnaire response rate was $55 \%$ (46 questionnaires returned out of 83 posted)

- $67 \%$ of operations were solely completed by the senior author and $33 \%$ by specialist registrar trainees under consultant supervision

- Eight revision cases (17\%) seven of which had their original surgery completed by other surgeons be that septorhinoplasty or solely septal surgery

- Majority of cases had a cartilage graft (80\%)

- Majority of patients (89\%) pre-operatively had a combination of nasal signs and symptoms namely, nasal obstruction, external nasal and septal distortion (reasserting the principle that ECS is reserved for difficult cases)

- External nasal deformities were either of cartilage pyramid deformities alone, or in combination with bony deformities +/- a dorsal hump.

- Nasal packing was performed in the majority of cases (78\%) usually dissolvable Nasopore ${ }^{\circledR}$ allowing for same day discharge.

- Standard follow-up was at 7-10 days for splint and suture removal and at 3 months for assessment.
- Minor complications consisted of an epistaxis post-surgery, which resolved with conservative treatment but delayed discharge and a pre-septal/facial cellulitis, which pre-operatively had a complex nasal deformity. The latter resolved with intravenous antibiotics.

- Two cases required further revision (i) secondary to an extruded PDS sheet and (ii) requiring a collumeloplasty for a hanging columellar. Another case was re-referred for assessment due to a recurrence of symptoms.

\section{Qualitative data}

A. Surgical Success

1) Does your nose feel more comfortable after the operation?

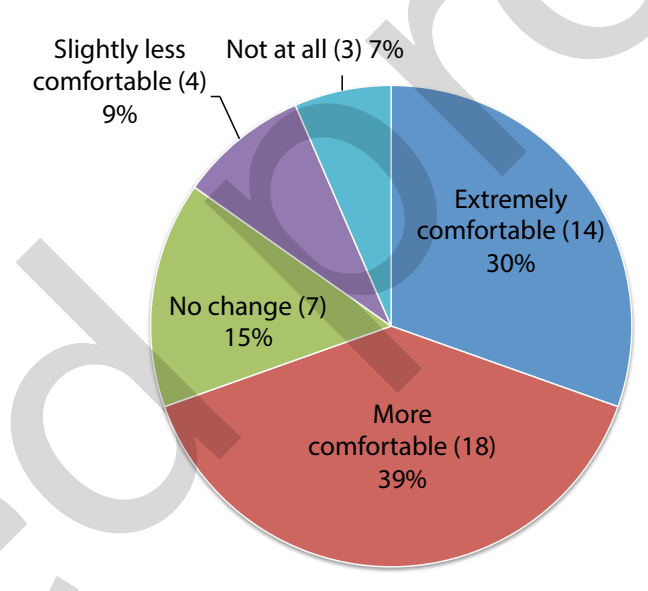

2) Was your nose blocked before the operation? Yes: $43(93 \%)$ versus $\quad$ No: $3(7 \%)$

2a) If so what degree of improvement in nasal obstruction has there been?

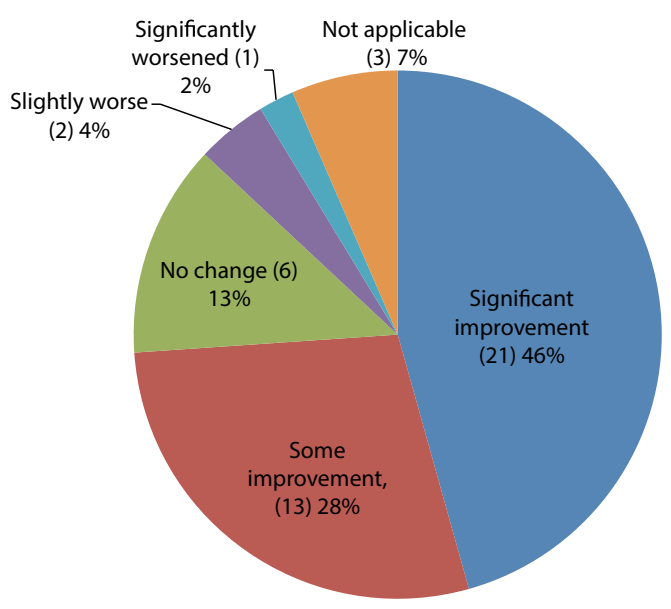

3) Do you have any other problems with your nose (allergy/ sinusitis)?

Yes: 15 (33\%) versus No: 31 (67\%) 
3a) If so what?

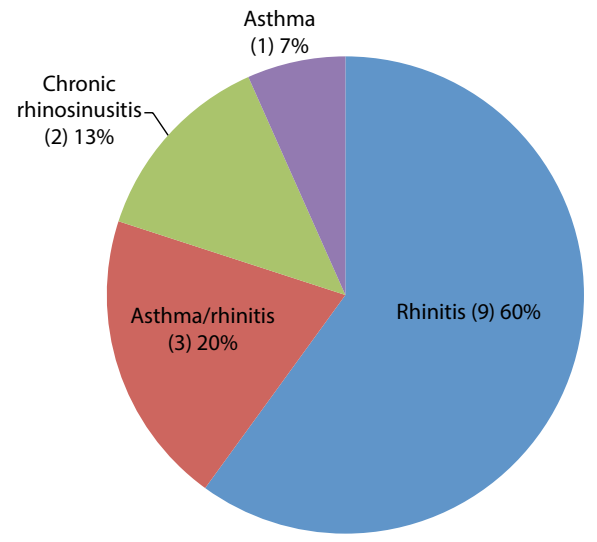

4) Do you feel the appearance of your nose has improved?

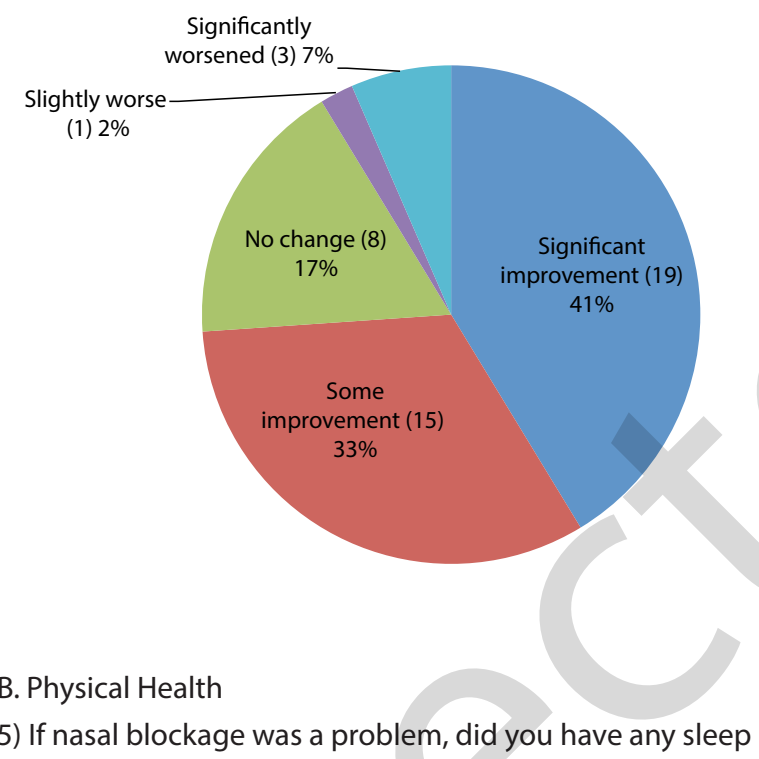

disturbance?

Yes: $26(57 \%)$ versus

No: 20 (43\%)

5a) If so, has your sleep improved after the operation?

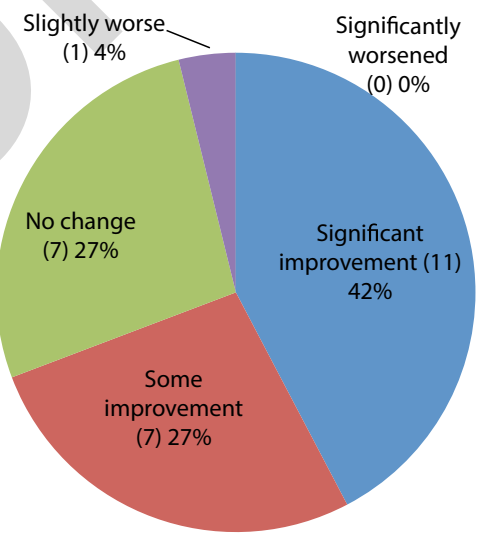

6) After the operation, do you feel better in yourself (health/well being)?

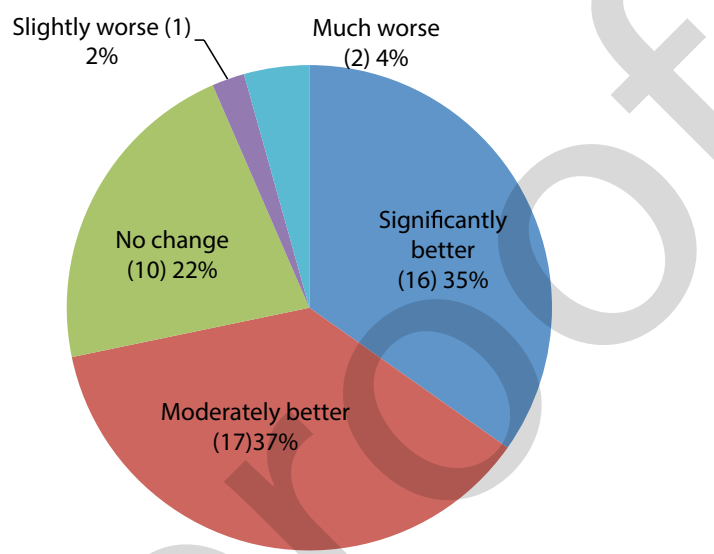

\section{Psychosocial Function}

7) Do you feel more or less self-conscious after the operation?

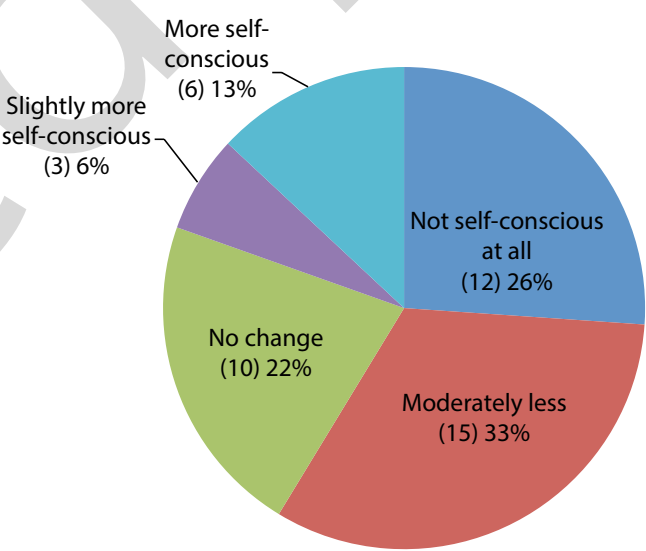

D. Social Interactions

8) Since your operation have you found it easier or harder to deal with company?

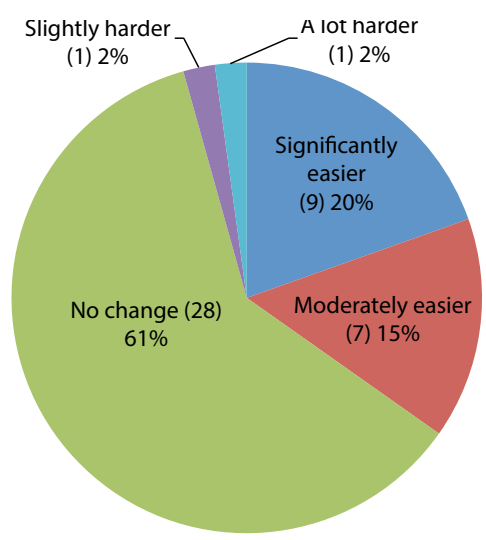


9) Do you have more or less self-confidence after the operation?

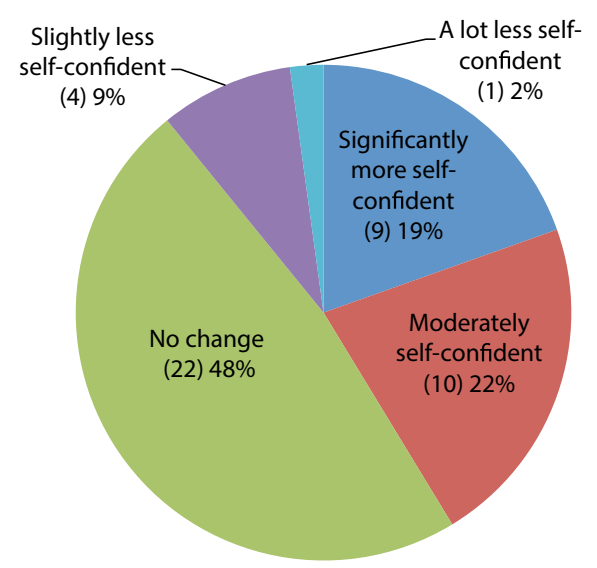

\section{Discussion}

The "twisted" nose poses difficulty due to the distortion and lateral displacement of the cartilaginous elements of the foundation tripod. There is usually an associated caudal dislocation or vertical fracture of the septum and irregularities of the tip in the form of spreading and loss of projection (the boxy tip), asymmetry or ptosis. The dorsum is held in its twisted shape by the rigidity of the junction between the upper lateral and the septal cartilages. Endonasal septoplasty cannot address this problem because the junction is not accessible via a closed approach. The open approach facilitates access to the septum and the upper lateral cartilages. The standard procedure is then to separate the upper laterals from the septum and centralise the latter in situ. The limitations here are twofold: firstly, it is difficult to deal with the dislocation or angulation of the septal cartilage in situ whilst creating a supportive and rigid enough structure to overcome the distortion and secondly, separating the upper laterals from the septum negates the natural "spreading" arrangement and risks internal valve obstruction. Spreader grafts may address this problem.

In the extracorporeal septorhinoplasty, the entire septal cartilage is disarticulated and separated flush from the upper laterals but preserving their attachment of the latter to one another thus avoiding middle third narrowing. The dorsum is thereby softened and its shape can be influenced by the septum in its new position. The intact middle third of the nose provides a firm base for supratip grafting if required. Once the foundation tripod has been stabilised, the caudal septum acts as support for tip reshaping and repositioning. The mobility of the tip will be compromised but it does not seem to create functional or aesthetic difficulty.

Our study demonstrates an improvement in patient satisfaction scores for the parameters of cosmetic appearance, comfort and nasal airway after surgery. Additional congestive factors such as (allergy/rhinitis) were present in a third of cases. In terms of physical health the majority of respondents reported feeling better in themselves with additional improvements in sleep quality in the $57 \%$ that presented with this symptom. The surgery did not markedly affect outcomes in psychosocial function or social interaction patterns with a significant proportion of respondents rating no change. This preliminary retrospective study demonstrates that in select patients with complex deformities, extracorporeal septorhinoplasty appears to offer high rates of patient satisfaction in terms of improvements in appearance, comfort, nasal airway and sleep quality.

A prospective study using the full Glasgow Benefit Inventory questionnaire status would be invaluable in assessing the patient-orientated post-interventional outcomes for extracorporeal septorhinoplasty, to compare its place in the gamut of septorhinoplasty techniques.

\section{Acknowledgement}

The authors would like to thank the secretarial staff and audit departments based at New Cross Hospital, Wolverhampton and Russell's Hall Hospital, Dudley for their help in this study.

\section{Authorship contribution}

DM: Data collection (auditor), data analysis, paper write-up and revisions. PPC: Data collection (auditor). LPG: Lead operating surgeon, audit supervisor, paper write-up and revisions.

\section{Conflict of interest}

None declared by the authors.

\section{References}

1. Robinson K, Gatehouse S, Browning GG. Measuring patient benefit from otorhinological surgery and therapy. Ann. Otol. Rhinolo. Laryngol. 1996; 105(6): 415-422.

2. King ED, Ashley FL. The correction of the internally and externally deviated nose. Plast. Reconstr. Surg. 1952; 10(2):116-120.

3. Rees TD. Surgical correction of the severely deviated nose by extramucosal excision of the osseocartilaginous septum and replacement as a free graft. Plast. Reconstr. Surg. 1986; 78(3): 320-330.
4. Gubisch W. The extracorporeal septum plasty: a technique to correct difficult nasal deformities. Plast. Reconstr. Surg. 1995; 95(4): 672-682.
Miss D. Mendis

Department of ENT Surgery

New Cross Hospital NHS Trust

Wednesfield Road

Wolverhampton WV10 0QP

United Kingdom

Tel: +44-(0)1902 307999

Fax: +44-(0)1902 695634

E-mail:dulanimendis@yahoo.com 